PROCEEDINGS OF THE

AMERICAN MATHEMATICAL SOCIETY

Volume 138, Number 12, December 2010, Pages 4167-4175

S 0002-9939(2010)10443-4

Article electronically published on May 28, 2010

\title{
A NOTE ON THE GAPS BETWEEN CONSECUTIVE ZEROS OF THE RIEMANN ZETA-FUNCTION
}

\author{
H. M. BUI, M. B. MILINOVICH, AND N. C. NG
}

(Communicated by Ken Ono)

\begin{abstract}
Assuming the Riemann Hypothesis, we show that infinitely often consecutive non-trivial zeros of the Riemann zeta-function differ by at most 0.5155 times the average spacing and that infinitely often they differ by at least 2.6950 times the average spacing.
\end{abstract}

\section{INTRODUCTION}

Let $\zeta(s)$ denote the Riemann zeta-function. Assuming the Riemann Hypothesis, the non-trivial zeros of $\zeta(s)$ can be written as $\rho=\frac{1}{2} \pm i \gamma$, where $\gamma$ is a positive real number. It is well known that, for $T \geq 10$,

$$
N(T):=\sum_{0<\gamma \leq T} 1=\frac{T}{2 \pi} \log \frac{T}{2 \pi}-\frac{T}{2 \pi}+O(\log T) .
$$

Hence, if we let $0<\gamma \leq \gamma^{\prime}$ denote consecutive ordinates of non-trivial zeros of $\zeta(s)$, we see that the average size of $\gamma^{\prime}-\gamma$ is $2 \pi / \log \gamma$. Normalizing, we let

$$
\lambda:=\limsup _{\gamma>0} \frac{\left(\gamma^{\prime}-\gamma\right) \log \gamma}{2 \pi}
$$

and

$$
\mu:=\liminf _{\gamma>0} \frac{\left(\gamma^{\prime}-\gamma\right) \log \gamma}{2 \pi},
$$

and we observe that $\mu \leq 1 \leq \lambda$. It is expected that there are arbitrarily large and arbitrarily small (normalized) gaps between consecutive zeros of the Riemann zeta-function on the critical line; in other words, that $\mu=0$ and $\lambda=+\infty$. In this paper, we prove the following theorem.

Theorem 1.1. Assume the Riemann Hypothesis. Then $\lambda>2.6950$ and $\mu<0.5155$.

We briefly describe the history of the problem. Very little is known unconditionally; however, Selberg (unpublished, but announced in 12]) has shown that $\mu<1<\lambda$. Assuming the Riemann Hypothesis, numerous authors 2, 5, 7, 8, 10,

Received by the editors September 5, 2009 and, in revised form, February 9, 2010.

2010 Mathematics Subject Classification. Primary 11M26; Secondary 11M06.

Key words and phrases. Distribution of zeros, Riemann Hypothesis, Riemann zeta-function.

The first author was supported by an EPSRC Postdoctoral Fellowship.

The second author was supported in part by a University of Mississippi College of Liberal Arts summer research grant.

The third author was supported in part by an NSERC Discovery grant.

(C)2010 American Mathematical Society Reverts to public domain 28 years from publication 
have obtained explicit bounds for $\mu$ and $\lambda$. Theorem 1.1 improves the previously best known results under this assumption which were $\mu<0.5172$ due to Conrey, Ghosh, and Gonek [2] and $\lambda>2.6306$ due to R. R. Hall [5]. The results in Hall's paper are actually unconditional, but a lower bound for $\lambda$ can only be obtained if the Riemann Hypothesis is assumed. Assuming the generalized Riemann Hypothesis for the zeros of Dirichlet $L$-functions, Conrey, Ghosh, and Gonek 3 have shown that $\lambda>2.68$. Their method can be modified (see [1] and [1) to show that $\lambda>3$.

Understanding the distribution of the zeros of the zeta-function is important for a number of reasons. One reason, in particular, is the connection between the spacing of the zeros of $\zeta(s)$ and the class number problem for imaginary quadratic fields. This is described by Conrey and Iwaniec in [4]; see also Montgomery and Weinberger [9]. Studying this connection led Montgomery [7] to investigate the pair correlation of the ordinates of the zeros of the zeta-function. He conjectured that, for any fixed $0<\alpha<\beta$,

$$
\sum_{\substack{0<\gamma, \tilde{\gamma} \leq T \\ \frac{2 \pi \alpha}{\log T} \leq \tilde{\gamma}-\gamma \leq \frac{2 \pi \beta}{\log T}}} 1 \sim N(T) \int_{\alpha}^{\beta}\left(1-\left(\frac{\sin \pi u}{\pi u}\right)^{2}\right) d u .
$$

Here $\gamma$ and $\tilde{\gamma}$ run over two distinct sets of ordinates of the non-trivial zeros of $\zeta(s)$. Clearly, Montgomery's conjecture implies that $\mu=0$. Moreover, F. J. Dyson observed that the eigenvalues of large, random complex Hermitian or unitary matrices have the same pair correlation function. This observation (among other things) has led to a stronger conjecture that the zeros of the zeta-function should behave, asymptotically, like the eigenvalues of large random matrices from the Gaussian Unitary Ensemble. These ideas led to the conjecture that $\lambda=+\infty$.

\section{Montgomery And OdLyzko's method For EXHibiting IRREgularity IN THE GAPS BETWEEN CONSECUTIVE ZEROS OF $\zeta(s)$}

Throughout the remainder of this paper, we assume the truth of the Riemann Hypothesis.

Let $T$ be large and put $K=T(\log T)^{-2}$. Further, let

$$
h(c):=c-\frac{\operatorname{Re}\left(\sum_{n k \leq K} a_{k} \overline{a_{n k}} g_{c}(n) \Lambda(n) n^{-1 / 2}\right)}{\sum_{k \leq K}\left|a_{k}\right|^{2}},
$$

where

$$
g_{c}(n)=\frac{2 \sin \left(\pi c \frac{\log n}{\log T}\right)}{\pi \log n}
$$

and $\Lambda(\cdot)$ is von Mangoldt's function defined by $\Lambda(n)=\log p$ if $n=p^{k}$ for a prime $p$ and $k \in \mathbb{N}$ and by $\Lambda(n)=0$ otherwise. In $[8]$, by an argument using the GuinandWeil explicit formula for the zeros of $\zeta(s)$, Montgomery and Odlyzko show that if $h(c)<1$ for some choice of $c>0$ and a sequence $\left\{a_{n}\right\}$, then $\lambda \geq c$ and that if $h(c)>1$ for a choice of $c>0$ and a sequence $\left\{a_{n}\right\}$, then $\mu \leq c$. In particular, for any such choices of $c$ and $\left\{a_{n}\right\}$, their method proves the existence of a pair of consecutive zeros of $\zeta(s)$ with ordinates $\gamma \leq \gamma^{\prime}$ in the interval $[T / 2,2 T]$ which satisfy $\gamma^{\prime}-\gamma \geq \frac{2 \pi c}{\log T}$ and $\gamma^{\prime}-\gamma \leq \frac{2 \pi c}{\log T}$, respectively. 
Conrey, Ghosh, and Gonek [2, expanding on an idea of Mueller [10, have given an alternate and much simpler way of viewing this problem. Let

$$
A(t)=\sum_{k \leq K} a_{k} k^{-i t}
$$

be a Dirichlet polynomial and set

$$
M_{1}=\int_{T / 2}^{2 T}|A(t)|^{2} d t \quad \text { and } \quad M_{2}(c)=\int_{-\pi c / \log T}^{\pi c / \log T} \sum_{T / 2 \leq \gamma \leq 2 T}|A(\gamma+\alpha)|^{2} d \alpha .
$$

Then, clearly, $M_{2}(c)$ is monotonically increasing and $M_{2}(\mu) \leq M_{1} \leq M_{2}(\lambda)$ when $T$ is sufficiently large. Therefore, if it can be shown that $M_{2}(c)<M_{1}$ for some choice of $A(t)$ and $c$, then $\lambda>c$. Similarly, if $M_{2}(c)>M_{1}$ for some choice of $A(t)$ and $c$, then $\mu<c$. Using standard techniques to estimate $M_{1}$ and $M_{2}(c)$, it can be shown that

$$
M_{2}(c) / M_{1}=h(c)+o(1) .
$$

Hence, this argument is seen to be equivalent to Montgomery and Odlyzko's method, described above. Moreover, we note that this formulation of the method suggests that we should choose a test function $A(t)$ which is small near the zeros of $\zeta(s)$ to exhibit large gaps between the zeros of the zeta-function and a test function $A(t)$ which is large near the zeros of $\zeta(s)$ to exhibit small gaps.

In [8], Montgomery and Odlyzko make the choices of

$$
a_{k}^{+}=\frac{1}{\sqrt{k}} f\left(\frac{\log k}{\log K}\right) \quad \text { and } \quad a_{k}^{-}=\frac{\lambda(k)}{\sqrt{k}} f\left(\frac{\log k}{\log K}\right)
$$

(using the coefficients $a_{k}^{+}$to exhibit large gaps and $a_{k}^{-}$to exhibit small gaps), where $f$ is a continuous function of bounded variation on $[0,1]$ normalized so that $\int_{0}^{1}|f|^{2}=1$ and $\lambda(k)$, the Liouville function, equals $(-1)^{\Omega(k)}$. Here, $\Omega(k)$ denotes the total number of primes dividing $k$. By choosing $f$ to be a certain modified Bessel function, the values $\mu<0.5179$ and $\lambda>1.9799$ are obtained. They mention that this choice of $f$ is nearly optimal for their choice of coefficients $\left\{a_{k}^{ \pm}\right\}$.

In 2, Conrey, Ghosh, and Gonek choose the coefficients

$$
a_{k}^{+}=\frac{d_{r}(k)}{\sqrt{k}} \quad \text { and } \quad a_{k}^{-}=\frac{\lambda(k) d_{r}(k)}{\sqrt{k}},
$$

where $d_{r}(k)$ is a multiplicative function defined on integral powers of a prime $p$ by

$$
d_{r}\left(p^{k}\right)=\frac{\Gamma(k+r)}{\Gamma(r) k !} .
$$

In this context, exhibiting large and small (normalized) gaps between consecutive zeros of the zeta-function becomes an optimization problem in the variable $r$. The choice $r=1.1$ yields $\mu<0.5172$, and the choice $r=2.2$ yields $\lambda>2.3378$.

In order to prove Theorem [1.1, we combine the approaches of [8] and [2]. We choose the coefficients

$$
a_{k}^{+}=\frac{d_{r}(k)}{\sqrt{k}} f\left(\frac{\log K / k}{\log K}\right) \quad \text { and } \quad a_{k}^{-}=\frac{\lambda(k) d_{r}(k)}{\sqrt{k}} f\left(\frac{\log K / k}{\log K}\right)
$$

for sufficiently smooth functions $f$. This variant allows us to optimize over both $r$ and $f$ rather than over just $r$ or just $f$. 
We now provide further insight into the choice of these coefficients. For simplicity, suppose $f$ is a polynomial. Since, for $\operatorname{Re} s>1$,

$$
\sum_{k \geq 1} \frac{d_{r}(k)}{k^{s}}=\zeta(s)^{r} \quad \text { and } \quad \sum_{k \geq 1} \frac{\lambda(k) d_{r}(k)}{k^{s}}=\left(\frac{\zeta(2 s)}{\zeta(s)}\right)^{r},
$$

with our choice of coefficients $\left\{a_{k}^{+}\right\}$and $\left\{a_{k}^{-}\right\}$we see that the test function $A(t)$ approximates $\zeta\left(\frac{1}{2}+i t\right)^{r}$ and $\zeta(1+2 i t)^{r} / \zeta\left(\frac{1}{2}+i t\right)^{r}$, respectively, and should have the desired effect of making $A(t)$ small (respectively large) near the zeros of $\zeta(s)$. Moreover, when we multiply $d_{r}(k)$ by $f\left(\frac{\log K / k}{\log K}\right)$, then $A(t)$ behaves like a linear combination of $\zeta\left(\frac{1}{2}+i t\right)^{r}$ and its derivatives and an analogous comment applies to the other case. The presence of the function $f$ leads to improved numerical results for bounds for $\mu$ and $\lambda$.

With the coefficients $\left\{a_{k}^{ \pm}\right\}$in (2.2), we define

$$
h^{ \pm}(c):=c-\frac{\operatorname{Re}\left(\sum_{n k \leq K} a_{k}^{ \pm} \overline{a_{n k}^{ \pm}} g_{c}(n) \Lambda(n) n^{-1 / 2}\right)}{\sum_{k \leq K}\left|a_{k}^{ \pm}\right|^{2}},
$$

where $g_{c}(n)$ is the arithmetic function defined in (2.1). In order to establish the bounds for $\lambda$ and $\mu$ in Theorem 1.1, we require the following lemma.

Lemma 2.1. Let $T$ be large, $K=T(\log T)^{-2}$, and $r \geq 1$. Then we have

$$
\begin{aligned}
h^{ \pm}(c)=c & \mp \frac{2 r}{\pi} \frac{\int_{0}^{1}(1-u)^{r^{2}-1} f(u) \int_{0}^{u} \frac{\sin (\pi c v)}{v} f(u-v) d v d u}{\int_{0}^{1}(1-u)^{r^{2}-1} f(u)^{2} d u} \\
& +O_{f, r, \varepsilon}\left((\log T)^{-1+\varepsilon}\right),
\end{aligned}
$$

where $f$ is a continuous, real-valued function of bounded variation on $L^{2}[0,1]$ and $\varepsilon>0$ is arbitrary.

We are now able to deduce Theorem 1.1 from Lemma 2.1 .

Proof of Theorem 1.1. We begin with the lower bound for $\lambda$. Choosing $r=3.00$ and

$$
f(x)=1+11 x+42 x^{2}+26 x^{3}-75 x^{4}
$$

in (2.3), a numerical calculation shows that $h^{+}(2.6950)<1$ when $T$ is sufficiently large. This provides the lower bound for $\lambda$ in Theorem 1.1

We now establish the upper bound for $\mu$. Choosing $r=1.23$ and

$$
f(x)=1+0.99 x-0.42 x^{2}
$$

in (2.3), a numerical calculation implies that $h^{-}(0.5155)>1$ for sufficiently large $T$. This provides the upper bound for $\mu$ stated in Theorem 1.1. (See Table 1 and Table 2 in $\S 3$ for some other numerically optimal choices of $f$.)

Our choices of $r$ and $f$ shall be explained in more detail in the next section. We conclude this section with the proof of Lemma 2.1.

Proof of Lemma 2.1. We begin by establishing the formula for $h^{+}(c)$ in (2.3). We assume that $r \geq 1$ so that $d_{r}(m n) \leq d_{r}(m) d_{r}(n)$ for $m, n \in \mathbb{N}$. It is well known that, for fixed $r \geq 1$,

$$
\sum_{k \leq x} \frac{d_{r}(k)^{2}}{k}=A_{r}(\log x)^{r^{2}}+O\left((\log T)^{r^{2}-1}\right)
$$


uniformly for $x \leq T$; here $A_{r}$ is a certain arithmetical constant (the exact value is not important in our argument). By partial summation, we find that the denominator in the ratio of sums in the definition of $h^{+}(c)$ is

$$
\begin{aligned}
\sum_{k \leq K}\left|a_{k}^{+}\right|^{2} & =\int_{1^{-}}^{K} f\left(\frac{\log K / x}{\log K}\right)^{2} d\left(\sum_{k \leq x} \frac{d_{r}(k)^{2}}{k}\right) \\
& =A_{r} r^{2} \int_{1}^{K} f\left(\frac{\log K / x}{\log K}\right)^{2}(\log x)^{r^{2}-1} \frac{d x}{x}+O_{f, r}\left((\log T)^{r^{2}-1}\right)
\end{aligned}
$$

by (2.4). By the variable change $u=1-\frac{\log x}{\log K}$, we have

$$
\sum_{k \leq K}\left|a_{k}^{+}\right|^{2}=A_{r} r^{2}(\log K)^{r^{2}} \int_{0}^{1}(1-u)^{r^{2}-1} f(u)^{2} d u+O_{f, r}\left((\log T)^{r^{2}-1}\right)
$$

where $\varepsilon>0$ is arbitrary.

We now evaluate the numerator in the ratio of sums in the definition of $h^{+}(c)$. If we let

$$
N^{+}(c):=\sum_{n k \leq K} a_{k}^{+} \overline{a_{n k}^{+}} g_{c}(n) \Lambda(n) n^{-1 / 2}
$$

then

$$
\begin{aligned}
N^{+}(c)= & \frac{2}{\pi} \sum_{n k \leq K} \frac{d_{r}(k) d_{r}(k n) \Lambda(n)}{k n \log n} f\left(\frac{\log K / k}{\log K}\right) f\left(\frac{\log K / n k}{\log K}\right) \sin \left(\pi c \frac{\log n}{\log T}\right) \\
= & \frac{2}{\pi} \sum_{p k \leq K} \frac{d_{r}(k) d_{r}(k p)}{k p} f\left(\frac{\log K / k}{\log K}\right) f\left(\frac{\log K / p k}{\log K}\right) \sin \left(\pi c \frac{\log p}{\log T}\right) \\
& \quad+O_{f, r}\left((\log T)^{r^{2}-1}\right) \\
= & \frac{2 r}{\pi} \sum_{p \leq K} \frac{\sin \left(\pi c \frac{\log p}{\log T}\right)}{p} \sum_{k \leq K / p} \frac{d_{r}(k)^{2}}{k} f\left(\frac{\log K / k}{\log K}\right) f\left(\frac{\log K / p k}{\log K}\right) \\
& \quad+O_{f, r}\left((\log T)^{r^{2}-1}\right),
\end{aligned}
$$

where the sum over $p$ runs over the primes. By Stieltjes' integration and a variable change, the inner sum in the main term of the last expression for $N^{+}(c)$ is

$$
\begin{array}{rl}
\int_{1^{-}}^{K / p} & f\left(\frac{\log K / x}{\log K}\right) f\left(\frac{\log K / p x}{\log K}\right) d\left(\sum_{k \leq x} \frac{d_{r}(k)^{2}}{k}\right) \\
= & A_{r} r^{2} \int_{1}^{K / p} f\left(\frac{\log K / x}{\log K}\right) f\left(\frac{\log K / p x}{\log K}\right)(\log x)^{r^{2}-1} \frac{d x}{x}+O_{f, r}\left((\log T)^{r^{2}-1}\right) \\
= & A_{r} r^{2}(\log K)^{r^{2}} \int_{\frac{\log p}{\log K}}^{1}(1-u)^{r^{2}-1} f(u) f\left(u-\frac{\log p}{\log K}\right) d u+O_{f, r}\left((\log T)^{r^{2}-1}\right) .
\end{array}
$$


By combining the above estimates and interchanging the order of summation and integration, we conclude that $N^{+}(c)=M^{+}(c)+O_{f, r}\left((\log T)^{r^{2}-1}\right)$, where

$$
\begin{aligned}
M^{+}(c)= & \frac{2 A_{r} r^{3}}{\pi}(\log K)^{r^{2}} \int_{\frac{\log 2}{\log K}}^{1}(1-u)^{r^{2}-1} f(u) \sum_{2 \leq p \leq K^{u}} \frac{\sin \left(\pi c \frac{\log p}{\log T}\right)}{p} f\left(u-\frac{\log p}{\log K}\right) d u \\
= & \left.\frac{2 A_{r} r^{3}}{\pi}(\log K)^{r^{2}} \int_{0}^{1}(1-u)^{r^{2}-1} f(u) \sum_{2 \leq p \leq K^{u}} \frac{\sin (\pi c \log p}{\log T}\right) \\
& +O_{f, r, \varepsilon}\left((\log T)^{r^{2}-1+\varepsilon}\right) .
\end{aligned}
$$

By the prime number theorem with remainder term, it follows that

$$
\sum_{2 \leq p \leq K^{u}} \frac{\sin \left(\pi c \frac{\log p}{\log T}\right)}{p} f\left(u-\frac{\log p}{\log K}\right)=\int_{2}^{K^{u}} \frac{\sin \left(\pi c \frac{\log x}{\log T}\right)}{x \log x} f\left(u-\frac{\log x}{\log K}\right) d x+O_{f, r}\left(\frac{1}{\log T}\right) .
$$

By the variable change $v=\frac{\log x}{\log K}$, the integral is

$$
\int_{\frac{\log 2}{\log K}}^{u} \frac{\sin \left(\pi c v \frac{\log K}{\log T}\right)}{v} f(u-v) d v=\int_{0}^{u} \frac{\sin (\pi c v)}{v} f(u-v) d v+O_{f, r, \varepsilon}\left((\log T)^{-1+\varepsilon}\right) .
$$

Hence,

$$
\begin{aligned}
N^{+}(c)= & \frac{2 A_{r} r^{3}}{\pi}(\log K)^{r^{2}} \int_{0}^{1}(1-u)^{r^{2}-1} f(u) \int_{0}^{u} \frac{\sin (\pi c v)}{v} f(u-v) d v d u \\
& +O_{f, r, \varepsilon}\left((\log T)^{r^{2}-1+\varepsilon}\right) .
\end{aligned}
$$

Combining our formulae for $\sum_{k \leq K}\left|a_{k}^{+}\right|^{2}$ and $N^{+}(c)$, we find that

$$
\begin{aligned}
h^{+}(c)=c & -\frac{2 r}{\pi} \frac{\int_{0}^{1}(1-u)^{r^{2}-1} f(u) \int_{0}^{u} \frac{\sin (\pi c v)}{v} f(u-v) d v d u}{\int_{0}^{1}(1-u)^{r^{2}-1} f(u)^{2} d u} \\
& +O_{f, r, \varepsilon}\left((\log T)^{-1+\varepsilon}\right),
\end{aligned}
$$

as claimed.

Since the proof of the formula for $h^{-}(c)$ is very similar to the proof of the formula for $h^{+}(c)$, we simply indicate the changes that need to be made in the above argument. In this case, we would consider the coefficients $a_{k}^{-}=\frac{\lambda(k) d_{r}(k)}{\sqrt{k}} f\left(\frac{\log K / k}{\log K}\right)$. Note that

$$
\lambda(n)^{2}=1 \text { and } \lambda(p n)=-\lambda(n)
$$

for every $n \in \mathbb{N}$ and every prime $p$. The first identity in (2.6) implies that

$$
\sum_{k \leq K}\left|a_{k}^{-}\right|^{2}=\sum_{k \leq K}\left|a_{k}^{+}\right|^{2}=\sum_{k \leq K} \frac{d_{r}(k)^{2}}{k} f\left(\frac{\log K / k}{\log K}\right)^{2},
$$

and using the second identity in (2.6) it is not hard to show that

$$
\begin{aligned}
\sum_{n k \leq K} a_{k}^{-} \overline{a_{n k}^{-}} g_{c}(n) \Lambda(n) n^{-1 / 2} & \\
=- & \frac{2}{\pi} \sum_{p k \leq K} \frac{d_{r}(k) d_{r}(k p)}{k p} f\left(\frac{\log K / k}{\log K}\right) f\left(\frac{\log K / p k}{\log K}\right) \sin \left(\pi c \frac{\log p}{\log T}\right) \\
& +O_{f, r}\left((\log T)^{r^{2}-1}\right) .
\end{aligned}
$$


Each of these expressions were dealt with in our evaluation of $h^{+}(c)$. The only difference is the - sign in the second identity. Thus, by the above calculations, we find that

$$
\begin{aligned}
h^{-}(c)=c & +\frac{2 r}{\pi} \frac{\int_{0}^{1}(1-u)^{r^{2}-1} f(u) \int_{0}^{u} \frac{\sin (\pi c v)}{v} f(u-v) d v d u}{\int_{0}^{1}(1-u)^{r^{2}-1} f(u)^{2} d u} \\
& +O_{f, r, \varepsilon}\left((\log T)^{-1+\varepsilon}\right) .
\end{aligned}
$$

This completes the proof of Lemma 2.1.

\section{Numerical CALCUlations}

In this section, we summarize the numerical calculations which led to Theorem 1.1. This theorem establishes the best known bounds for $\lambda$ and $\mu$ assuming the Riemann Hypothesis; however, we are still far from proving the conjectured values of $\mu=0$ and $\lambda=\infty$. In fact, it is known that this is not attainable using Montgomery and Odlyzko's method with Dirichlet polynomials of length $\leq T$. Specifically, in [2], it is shown that $h(c)<1$ if $c<\frac{1}{2}$ and $h(c)>1$ if $c \geq 6.2$. Moreover, the authors note, without proof, that $h(c)>1$ if $c \geq 3.74$. It would be interesting to better understand the limitations of this method and, in particular, if it can be used to show that $\mu \leq \frac{1}{2}$.

We have not been able to prove that our bounds for $\lambda$ and $\mu$ in Theorem 1.1 are the optimal bounds for our choice of coefficients $\left\{a_{k}^{ \pm}\right\}$in (2.2). In the special case of $r=1$, this optimization problem has been solved (in terms of prolate spheroidal wave functions). See comments in [8] and the articles [13] and [6]. When $r \neq 1$, the analogous optimization problem seems considerably more difficult. Instead of trying to solve it explicitly, we have instead chosen $f$ to be a polynomial of low degree $(\leq 6)$ having the form $f(x)=1+\alpha_{1} x+\alpha_{2} x^{2}+\cdots+\alpha_{k} x^{k}$. Using Mathematica, we numerically evaluated (2.5) and (2.7) for each choice of $c$ and $r$ in terms of the coefficients $\alpha_{1}, \alpha_{2}, \ldots, \alpha_{k}$. Then, using the Minimize/Maximize commands, we were able to find numerically optimal polynomials of each degree. Our results are summarized in the following tables. The coefficients of the polynomials in Table 1 are rounded to the nearest integer, and the coefficients in Table 2 are rounded to two significant figures.

TABLE 1. Using the coefficients $\left\{a_{k}^{+}\right\}$defined in (2.2), the following table displays some numerically optimal polynomials of low degree for which $h^{+}(c)<1$.

\begin{tabular}{|c||ccc|}
\hline Degree & Value of $c$ & Value of $r$ & Polynomial \\
\hline \hline 0 & 2.3378 & 2.17 & 1 \\
1 & 2.6779 & 2.87 & $1+30 x$ \\
2 & 2.6938 & 3.02 & $1+14 x+39 x^{2}$ \\
3 & 2.6949 & 3.00 & $1+9 x+60 x^{2}-45 x^{3}$ \\
4 & 2.6950 & 3.00 & $1+11 x+42 x^{2}+26 x^{3}-75 x^{4}$ \\
5 & 2.6950 & 3.00 & $1+12 x+35 x^{2}+61 x^{3}-155 x^{4}+60 x^{5}$ \\
6 & 2.6950 & 3.00 & $1+12 x+39 x^{2}+37 x^{3}-67 x^{4}-77 x^{5}+76 x^{6}$ \\
\hline
\end{tabular}


TABLE 2. Using the coefficients $\left\{a_{k}^{-}\right\}$defined in (2.2), the following table displays some numerically optimal polynomials of low degree for which $h^{-}(c)>1$.

\begin{tabular}{|c||ccc|}
\hline Degree & Value of $c$ & Value of $r$ & Polynomial \\
\hline \hline 0 & .5172 & 1.1 & 1 \\
1 & .5156 & 1.23 & $1+0.59 x$ \\
2 & .5155 & 1.23 & $1+0.99 x-0.42 x^{2}$ \\
3 & .5155 & 1.23 & $1+0.9 x-0.19 x^{2}-0.16 x^{3}$ \\
\hline
\end{tabular}

Our numerical calculations seem to suggest that polynomials of low degree polynomials work well; it does not seem like there is much to gain by taking $f$ to be a polynomial of degree greater than 4 . To demonstrate this phenomenon, we observe that one can recover the bounds for $\lambda$ and $\mu$, in the case of $r=1$, derived in [8] using polynomials of low degree in place of the modified Bessel functions. Letting $f(x)=1+6.47 x+15.36 x^{2}-43.65 x^{3}+21.83 x^{4}$, a numerical calculation shows that $h^{+}(1.9799)<1$, and if we let $f(x)=1+0.465 x-0.465 x^{2}$, then it can be shown that $h^{-}(0.5179)>1$. These are the nearly optimal values obtained by Mongomery and Odlyzko in [8] when $r=1$.

From Table 1 it appears that the optimal value that can be obtained for $\lambda$ occurs when $r \approx 3$. It should be noted that $r=3$ does not appear to give the optimal value, as we are able to show that $r=2.998$ gives a slightly better value for $\lambda$ using polynomials of low degree. It would be interesting to determine, in the spirit of the articles [13] and [6], the choices of $r$ and $f$ which give the optimal values for $\lambda$ and $\mu$.

\section{ACKNOWLEDGEMENT}

The authors would like to thank the anonymous referee for a number of helpful suggestions.

\section{REFERENCES}

1. H. M. Bui, Large gaps between consecutive zeros of the Riemann zeta-function, submitted. Available on the arXiv at http://arxiv.org/abs/0903.4007.

2. J. B. Conrey, A. Ghosh, and S. M. Gonek, A note on gaps between zeros of the zeta function, Bull. London Math. Soc. 16 (1984), 421-424. MR749453 (86i:11048)

3. J. B. Conrey, A. Ghosh, and S. M. Gonek, Large gaps between zeros of the zeta-function, Mathematika 33 (1986), 212-238. MR882495 (88g:11057)

4. J. B. Conrey and H. Iwaniec, Spacing of zeros of Hecke L-functions and the class number problem, Acta Arith. 103 (2002), no. 3, 259-312. MR.1905090 (2003h:11103)

5. R. R. Hall, A new unconditional result about large spaces between between zeta zeros, Mathematika 52 (2005), 101-113. MR2261847 (2007g:11104)

6. H. J. Landau and H. O. Pollak, Prolate spheroidal wave functions, Fourier analysis and uncertainty. II, Bell System Tech. J. 40 1961, 65-84. MR0140733 (25:4147)

7. H. L. Montgomery, The pair correlation of the zeros of the zeta function, Proc. Symp. Pure Math. 24, A.M.S., Providence, RI, 1973, 181-193. MR.0337821 (49:2590)

8. H. L. Montgomery and A. M. Odlyzko, Gaps between zeros of the zeta function, Colloq. Math. Soc. Jānos Bolyai, 34. Topics in Classical Number Theory (Budapest, 1981), North-Holland, Amsterdam, 1984. MR0781177(86e:11072) 
9. H. L. Montgomery and P. J. Weinberger, Notes on small class numbers, Acta Arith. 24 (1974), 529-542. MR0357373 (50:9841)

10. J. Mueller, On the difference between consecutive zeros of the Riemann zeta-function, J. Number Theory 14 (1983), 393-396. MR660377 (83k:10074)

11. N. Ng, Large gaps between the zeros of the Riemann zeta function, J. Number Theory 128 (2008), 509-556. MR2389854(2008m:11176)

12. A. Selberg, The zeta-function and the Riemann Hypothesis, Skandinaviske Matematikerkongres 10 (1946), 187-200. MR0019676 (8:446i)

13. D. Slepian and H. O. Pollak, Prolate spheroidal wave functions, Fourier analysis and uncertainty, I. Bell System Tech. J. 40 1961, 43-63. MR0140732 (25:4146)

Mathematical Institute, University of Oxford, Oxford, OX1 3LB United Kingdom

E-mail address: hung.bui@maths.ox.ac.uk

Department of Mathematics, University of Mississippi, University, Mississippi 38677

E-mail address: mbmilino@olemiss.edu

Department of Mathematics and Computer Science, University of Lethbridge, LethBRIDGE, AB, CANADA T1K 3M4

E-mail address: ng@cs.uleth.ca 